\title{
Ultra-high-speed digital in-line holography system applied to particle-laden supersonic underexpanded jet ows
}

\author{
Ingvorsen, Kristian Mark; Buchmann, Nicolas A.; Soria, Julio
}

Published in:

28th AIAA Aerodynamic Measurement Technology, Ground Testing, and Flight Testing Conference

Publication date:

2012

Link back to DTU Orbit

Citation (APA):

Ingvorsen, K. M., Buchmann, N. A., \& Soria, J. (2012). Ultra-high-speed digital in-line holography system applied to particle-laden supersonic underexpanded jet ows. In 28th AIAA Aerodynamic Measurement Technology, Ground Testing, and Flight Testing Conference American Institute of Aeronautics and Astronautics.

\section{General rights}

Copyright and moral rights for the publications made accessible in the public portal are retained by the authors and/or other copyright owners and it is a condition of accessing publications that users recognise and abide by the legal requirements associated with these rights.

- Users may download and print one copy of any publication from the public portal for the purpose of private study or research.

- You may not further distribute the material or use it for any profit-making activity or commercial gain

- You may freely distribute the URL identifying the publication in the public portal 


\title{
Ultra-high-speed digital in-line holography system applied to particle-laden supersonic underexpanded jet flows
}

\author{
Kristian M. Ingvorsen* \\ Technical University of Denmark, Kgs. Lyngby, 2800, Denmark, \\ Monash University, Melbourne, VIC, 3800, Australia \\ Nicolas A. Buchmann ${ }^{\dagger}$ and Julio Soria ${ }^{\ddagger}$ \\ Monash University, Melbourne, VIC, 3800, Australia
}

\begin{abstract}
Particle-fluid interactions in supersonic flows are relevant in many different applications e.g. the cold gas-dynamic spray process. The optimal application of the process is hindered by a lack of understanding of the particle-fluid interactions. To obtain detailed information on the particle-fluid interactions in these high-speed flows special high performance techniques are required. The present work is an investigation into the applicability of magnified digital in-line holography with ultra-high-speed recording for the study of three-dimensional supersonic particle-laden flows.

An optical setup for magnified digital in-line holography is created, using an ultrahigh-speed camera capable of frame rates of up to $1.0 \mathrm{MHz}$. To test the new technique an axisymmetric supersonic underexpanded particle-laden jet is investigated. The results show that the new technique allows for the acquisition of time resolved qualitative and quantitative data on the fluid and particle dynamics.

The potential for determining the three-dimensional particle positions using holographic reconstruction is also investigated. Five different focus functions used for depthwise location are tested using two different planar targets, a $10 \times 10 \mathrm{~mm}$ calibration grid and $120 \mu \mathrm{m}$ particles on a glass plate. In the case with the calibration grid it is found that accurate determination of the depthwise position is possible. However, when applying the same technique to the particle target, significant problems are encountered.
\end{abstract}

\section{Nomenclature}

$D \quad$ Nozzle exit diameter

$d_{p} \quad$ Mean particle diameter

NPR Nozzle pressure ratio

$P_{0} \quad$ Static pressure in settling chamber

$P_{\infty} \quad$ Ambient pressure

$x, y, z \quad$ Cartesian coordinates

$\rho_{f} \quad$ Fluid density

$\rho_{p} \quad$ Particle density

$n \quad$ Refractive index

$K \quad$ Gladstone-Dale constant

I Light intensity

${ }^{*}$ Ph.D. Student, Section of Fluid Mechanics, Coastal and Maritime Engineering, Department of Mechanical Engineering, Nils Koppels Allé Building 403, Kgs. Lyngby, 2800, Denmark, Non-member.

${ }^{\dagger}$ Research Fellow, Laboratory for Turbulence Research in Aerospace \& Combustion, Department of Mechanical \& Aerospace Engineering, Monash University, Melbourne VIC 3800 Australia, AIAA Member.

${ }^{\ddagger}$ Professor, Laboratory for Turbulence Research in Aerospace \& Combustion, Department of Mechanical \& Aerospace Engineering, Monash University, Melbourne VIC 3800 Australia, and AIAA Member. 


\section{Introduction}

Particle-fluid interactions in particle-laden supersonic jets are relevant in many different applications such as e.g. rocket motors, particle impactors, ${ }^{1}$ needle-free drug delivery devices ${ }^{2}$ and cold gas-dynamic spray processes. ${ }^{3}$ In the cold gas-dynamic spray process, small particles $(1-50 \mu \mathrm{m})$ in solid state are accelerated to high velocities $(300-1200 \mathrm{~m} / \mathrm{s})$ by a gas jet, and upon impaction against a substrate, form a deposit or coating. The process is thus characterized by a particle-laden supersonic jet. The jet flow is three dimensional unsteady and turbulent with complex shock structures embedded. The cold gas-dynamic spray process has several advantages compared to conventional coating technique, however, the optimal application of this techniques is hindered by a lack of understanding of the supersonic particle-laden jet, such as particle impact velocity and impact angle. Experimental data are needed to aid the understanding of the complex fluid dynamical processes but also to serve as validation material for numerical models. However, no experimental techniques are currently available that can deliver the detailed information needed. The main reason for this is that it is exceedingly difficult to measure the high-speed process due to the large dimensional gap between the very small particles $\left(\mathcal{O}\left(10^{-5}\right) \mathrm{m}\right)$, relatively large regions of interest $\left(\mathcal{O}\left(10^{-1}\right) \mathrm{m}\right)$ and very large velocities $\left(\mathcal{O}\left(10^{3}\right) \mathrm{m} / \mathrm{s}\right)$.

To illustrate the difficulty, the camera requirements for a particle tracking measurement are estimated by defining two simple criteria. (1) The exposure time must be small enough to effectively freeze the particles during recording and (2) the frame rate must be high enough to ensure a moderate particle displacement between frames. The maximum exposure time and minimum frame rate are evaluated (Fig. 1) for a nozzle exit diameter of $D=2 \mathrm{~mm}$ and three different particle diameters $d_{p}$ by requiring that the particle displacement during a single image exposure is limited to $d_{p}$ and that the displacement between frames is $D / 4$. From the figure it is seen that even for a large particle with moderate velocity $\left(d_{p}=100 \mu \mathrm{m}, u_{p}=200 \mathrm{~m} / \mathrm{s}\right)$, an exposure time of $0.50 \mu \mathrm{s}$ or less and a frame rate of $400 \mathrm{kHz}$ or above is needed, thus emphasizing the need for high performance equipment and techniques. In table I specifications for a standard camera, a highspeed camera, and an ultra-high-speed camera are presented. It should be noted that the spatial resolution is sacrificed for the increase in temporal resolution.
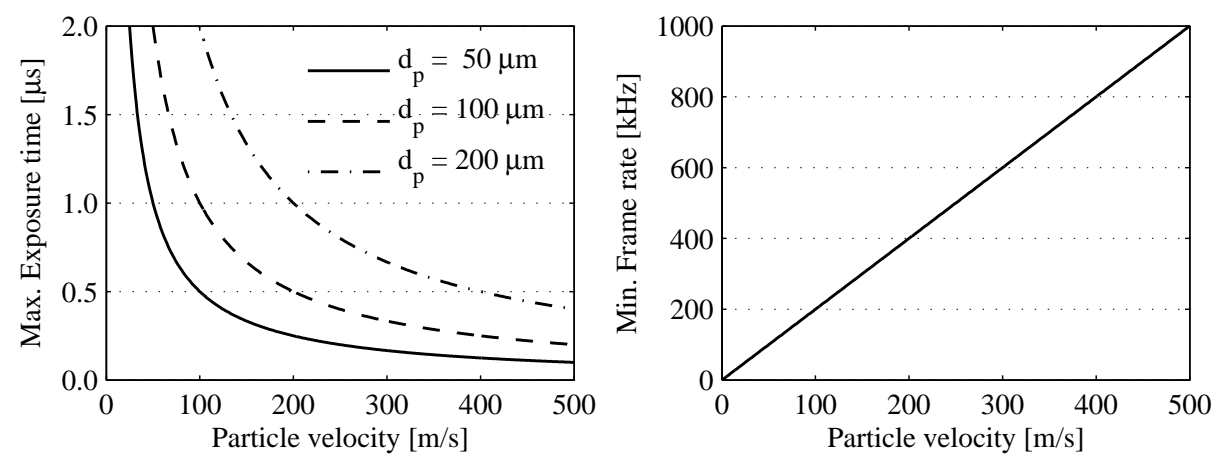

Figure 1. Example of camera requirements for a particle tracking measurement of a particle-laden supersonic jet

Table 1. Specifications for a standard camera, a high-speed camera and an ultra-high-speed camera

\begin{tabular}{lrrr}
\hline & $\begin{array}{r}\text { Chip size } \\
{[\mathrm{px} \times \mathrm{px}]}\end{array}$ & $\begin{array}{r}\text { Resolution } \\
{[\mu \mathrm{m} / \text { pixel }]}\end{array}$ & $\begin{array}{r}\text { frame rate } \\
{[\mathrm{Hz}]}\end{array}$ \\
\hline pco.2000 s & $2048 \times 2048$ & 7.4 & 8.2 \\
pco.dimax & $720 \times 480$ & 11.0 & 11,576 \\
Shimadzu HPV-1 & $312 \times 260$ & 66.3 & $1,000,000$ \\
\hline
\end{tabular}

The imaging of micron sized particles moving in 3D volumes creates a series of problems. ${ }^{4}$ Digital recording media such as CCD chips have a relatively low spatial resolution compared to the particle size. 
The low resolution of the recording media can be overcome by applying high magnification. However, for classical imaging techniques this results in a limited field of view and more critically a strongly reduced depth of field. One way to reduce the problem of limited depth of field is to use holographic techniques such as magnified or microscopic digital in-line holography. ${ }^{4,5}$ Although the technique struggles with the resolution of the digital recording media, the approach has successfully been used to locate and track slow moving particles in three dimensional volumes. ${ }^{4-6}$

However, when applying magnified in-line holography to a supersonic flow two additional issues arise. The first issue is that the spatial resolution of the ultra-high-speed camera is significantly lower than that of a standard CCD camera, worsening the problem for the holographic method. Secondly, the fluid density $\rho_{f}$ of a supersonic jet varies significantly in space. The fluid density is related to the refractive index $n$ by the Clasusius-Mosotti equation which for a gas reduces to the Gladstone-Dale equation given by $n-1=K \rho_{f}$, where $K$ is the Gladstone-Dale constant. The variation of refractive index through the jet causes the light rays to deflect thereby disturbing the laser beam. A visualization of the density field of the jet, similar to that obtained by shadowgraphy, is therefore overlain on the holograms. Despite this, the magnified digital in-line holographic setup still has great potential compared to traditional techniques when it comes to a flow such as a supersonic particle laden jet. The main advantages being that the depth of field problem is avoided and that the holographic techniques can give the three-dimensional position of the particles. Furthermore, by combining time resolved holographic measurements with 3D particle image velocimetry cross-correlation techniques it is possible to extract the three component particle velocity.

The present work is an investigations into the applicability of magnified digital in-line holographic with ultra-high-speed recording for the study of three dimensional supersonic particle-laden flows. In order to test the technique an axisymmetric particle-laden underexpanded supersonic jet is investigated. The results show that the new technique allows for the acquisition of both qualitative data (visualizations) and quantitative data (in-plane particle tracking 2D-2C, not included in this paper) on the fluid and particle dynamics. Lastly the potential for accurate determination of the depthwise position of objects in three dimensions are investigated using planar test targets. Two targets are used, the fist being a $10 \times 10 \mathrm{~mm}$ calibration grid and the second is $120 \mu \mathrm{m}$ particles on a glass plate. In the case with the grid it is found that accurate location is possible, while significant problems are encountered when trying to locate the particles on the glass plate.

\section{Experimental Methodology}

\section{II.A. Optical setup}

A schematic of the optical setup for the magnified digital in-line holography system is presented in Fig. 2. The illumination consist of a $200 \mathrm{~mW}$ continuous wave diode pumped laser (CrystaLaser CL532-200-S) with a wavelength of $532 \mathrm{~nm}$, a beam diameter of $0.36 \mathrm{~mm}$ and a coherence length of more than $300 \mathrm{~m}$. A neutral density (ND) filter wheel is mounted after the laser to allow for adjustment of the beam power. The laser beam is then expanded and collimated using three lenses $\left(L_{1}, L_{2}, L_{3}\right) . L_{1}$ and $L_{2}$ are plano concave lenses with focal lengths of $f=-30 \mathrm{~mm}$ and $f=-50 \mathrm{~mm}$, respectively and $L_{3}$ is a double convex lens with a focal length of $f=250 \mathrm{~mm}$. This configuration allows for an adjustable expansion factor and a very compact optical setup. The diameter of the expanded and collimated laser beam is approximately $35 \mathrm{~mm}$.

The imaging system consist of a $85 \mathrm{~mm}$ lens (Nikkon Nikkor) mounted to the camera using bellows. To increase the magnification further a 1:2 teleconverter is inserted between the bellows and the camera. The camera is an ultra-high-speed 10-bit camera (Shimadzu HPV-1) capable of recording 102 consecutive images with a frame rate of up to $1.0 \mathrm{MHz}$. The camera chip has a resolution of $312 \mathrm{px} \times 260 \mathrm{px}$ and a pixel pitch of $66.3 \mu \mathrm{m}$. The main features of the lens-bellows assembly are that it gives an adjustable magnification and secondly it results in a focal plane located approximately $100 \mathrm{~mm}$ in front of the lens $\left(L_{4}\right)$. The fact that the focal plane is located a significant distance from the camera is an advantage as it ensures that the flow remains undisturbed whilst at the same time minimizes the risk of getting particles on the lens. The exact magnification factor of the system is determined by recording an image of a $10 \mathrm{~mm} \times 10 \mathrm{~mm}$ calibration grid. For the presented data the magnification is 3.90 which corresponds to a resolution of $17.0 \mu \mathrm{m} /$ pixel and a field of view of $2.65 D \times 2.21 D$, where $D=2.0 \mathrm{~mm}$ is the nozzle exit diameter (c.f. section II.B). The camera is mounted such that the long side of the field of view is oriented in the streamwise direction.

The imaging system (lens, bellows and camera) is mounted on an optical rail allowing it to be traversed along the optical axis making it possible to change the position of the focal plane. The imaging system can be used in two different modes of operation depending on the position of the focal plane. In the first 


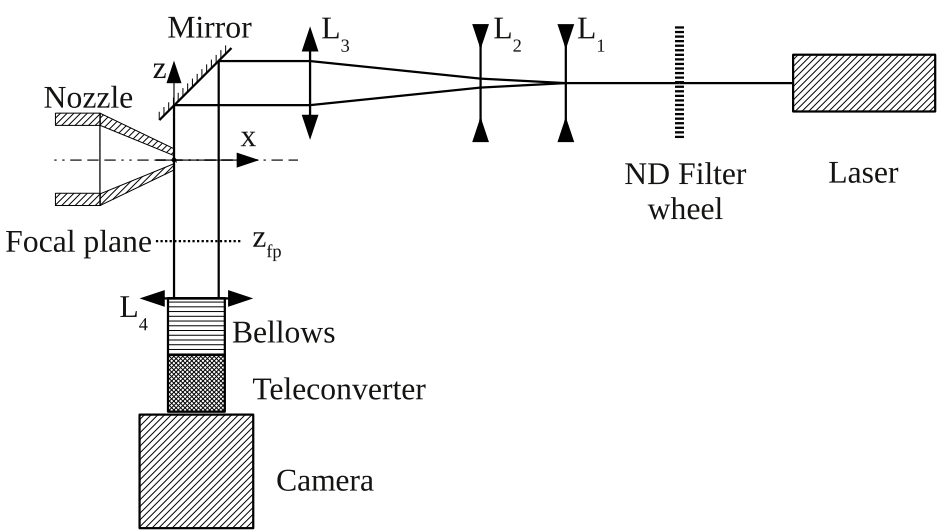

Figure 2. Schematic of the optical setup

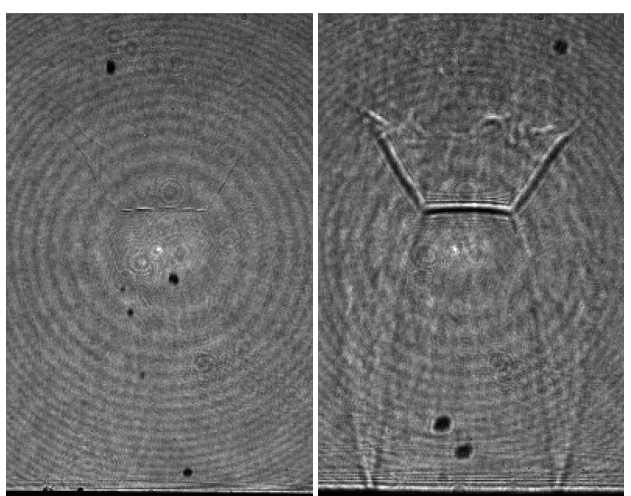

Figure 3. Different modes of the imaging system. Left: Shadow imaging. Right: Holography

mode, referred to as shadow imaging, the focal plane is located directly on the nozzle axis $\left(z_{f p}=0\right)$. In this mode the imaging system has a very low sensitivity to variations in the density field making it possible to visualize the particles without additional disturbances. The particles are clearly visualized by their shadow, allowing their size and shape to be estimated. Visualizations obtained using this mode is well suited for two dimensional particle tracking algorithms. Compared to traditional imaging techniques, the effective depth of field of shadow imaging is significantly increased. In the second mode, referred to as holographic mode, the focal plane is located behind the jet axis $\left(z_{f p}<0\right)$. In this configuration the system will record the hologram of the suspended particles with the visualization of the density field overlain. These recordings are well suited for qualitative visualizations of the interactions of the particles and the flow. Examples of images recorded using the two different modes of recording are presented in Fig. 3.

\section{II.B. Flow rig}

For the present work a flow rig has been developed that allows for controlled injection of particles into a convergent nozzle (Fig. 4). The nozzle exit diameter is $D=2 \mathrm{~mm}$ with a nozzle area ratio of 400 . The jet flow is characterized using the nozzle pressure ratio $\mathrm{NPR}=P_{0} / P_{\infty}$, where $P_{0}$ is the static pressure in the settling chamber, and $P_{\infty}$ is the ambient pressure. Compressed air is supplied to the nozzle through two separate supply lines. The main line, responsible for supplying the majority of the jets mass flow, consist of a pressure regulator and a manifold. The secondary line consists of a second pressure regulator and an entrainment chamber where the particles are suspended in the flow before being carried to the nozzle.

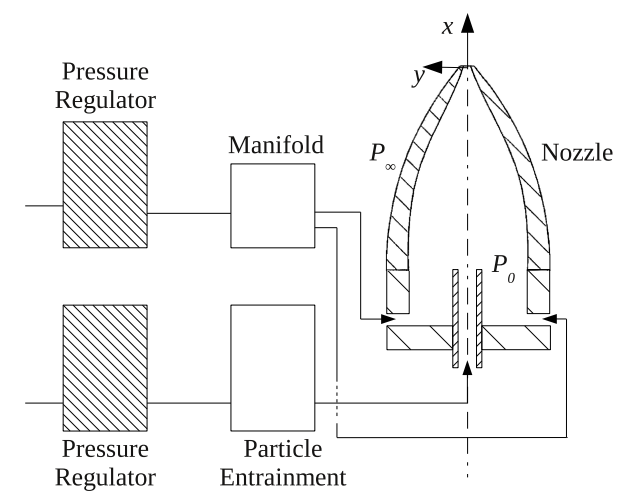

Figure 4. Schematic of the flow rig

Two particle types, both in the form of nylon powders, are used in the present work. The particles have volume weighted mean diameters of $d_{p}=57 \mu \mathrm{m}$ and $d_{p}=120 \mu \mathrm{m}$ (Vestosint 2157 and 1301, respectively). Additional details on the powders are given in Table 2. 
Table 2. Volume weighted mean diameter $d_{p}, 10 \%$ - and $90 \%$-percentiles of the particle size distribution and particle density $\rho_{p}$

\begin{tabular}{rrrr}
\hline$d_{p}[\mu \mathrm{m}]$ & $p_{10 \%}[\mu \mathrm{m}]$ & $p_{90 \%}[\mu \mathrm{m}]$ & $\rho_{p}\left[\mathrm{~kg} / \mathrm{cm}^{3}\right]$ \\
\hline 57 & 38 & 77 & 1.02 \\
120 & 64 & 188 & 1.06 \\
\hline
\end{tabular}

\section{Visualization of particle-flow interactions}

Qualitative information on the particle-fluid interaction in the supersonic particle-laden jet can be obtained from visualizations recorded using the holographic mode. For this purpose the focal plane of the system is located behind the nozzle axis $\left(z_{f p}=-7.5 \mathrm{~mm}\right)$, in order to be sensitive to the variation in the density field of the fluid. Figure 5 and 6 shows examples of visualizations recorded using the $d_{p}=120 \mu \mathrm{m}$ particles at two different NPRs. The contrast of the images has been adjusted in order to enhance the variation in the density field. It should be noted that the contrast adjustment makes the particle diffraction patterns appear as solid black particles. The actual particles are therefore smaller and in general more irregularly shaped then the presented diffraction patterns.

A sequence of 24 consecutive images of the particle-laden jet at NPR $=5.0$ recorded at $0.5 \mathrm{MHz}$ are shown in Fig. 5. The first frame of the series shows a relatively large particle located just upstream the Mach disc (a). It is observed that a bow shock is attached to the particle, indicating that the relative velocity, or slip velocity, between the particle and fluid is above Mach 1 . The bow shock disappears as soon as the particle has moved through the Mach disk as the fluid velocity is significant decreased over the Mach disk. For positions downstream the Mach disk the fluid rapidly accelerates again while the large inertia makes the particle lag behind resulting in the reappearance of the bow shock. In the first frame it is also seen that the particle has a significant downstream influence as a secondary shock appears behind the Mach disk (b). In the following frames it can be observed how the secondary shock moves downstream together with the particle and then disappears just as the particle has moved through the Mach disk. It is further observed that when the particle is in the immediate vicinity of the Mach disk (c), it significantly alters the shape of the shock structure (both Mach disk and oblique shock). The Mach disk and oblique shock return to their unperturbed shaped quickly after the particle has moved through the Mach disk. In the middle of the sequence a smaller particle exits the left side of the nozzle near the jet boundary (d). In the following frames it observed how the particle significantly interacts with the incidence, or barrel shock. The interaction results in the occurrence of two new shocks that are almost parallel, one attached to the upstream side of the particle and one located downstream the particle (e). In the last row of the sequence it is observed that two relatively large particles exits the nozzle close to each other. As the particles move away from the nozzle, the particle bow shocks are formed. It is noted that the bow shock from the upstream particle coincides with the location of the downstream particle (f). The behavior of the downstream particle is therefore expected to be affected by the presence of the upstream particle.

In a second example (Fig. 6) a single particle is shown passing through the Mach disk of a jet with $\mathrm{NPR}=6.5$. The sequence consist of 16 consecutive images recorded at a frame rate of $1.0 \mathrm{MHz}$. In the first frame the particle is located a distance upstream the Mach disk and a weak bow shock can be identified (a). In frame 6 the particle has moved closer to the Mach disk and a new shock structure has appeared (b). The structure is visualized by two oblique lines going from the downstream side of the particle to the Mach disk. In the following frame the two lines have evolved into a shock curving around the particle. In frame 8 the curved shock has fully connected to the oblique shock and forms one coherent structure. The Mach disk does however still exist and it appears that the curved shock and the Mach disk are of similar strength. In the remaining frames it is observed how the particle passes through the Mach disk, while the curved shock and the Mach disk merges.

\section{Holographic Reconstruction and Depth location}

In digital in-line holography, the interference pattern between the coherent reference beam and light diffracted by an object is recorded on the digital recording media. The recorded interference pattern contains information on both phase and amplitude of the recorded wave front, and it is thus possible to numerically 


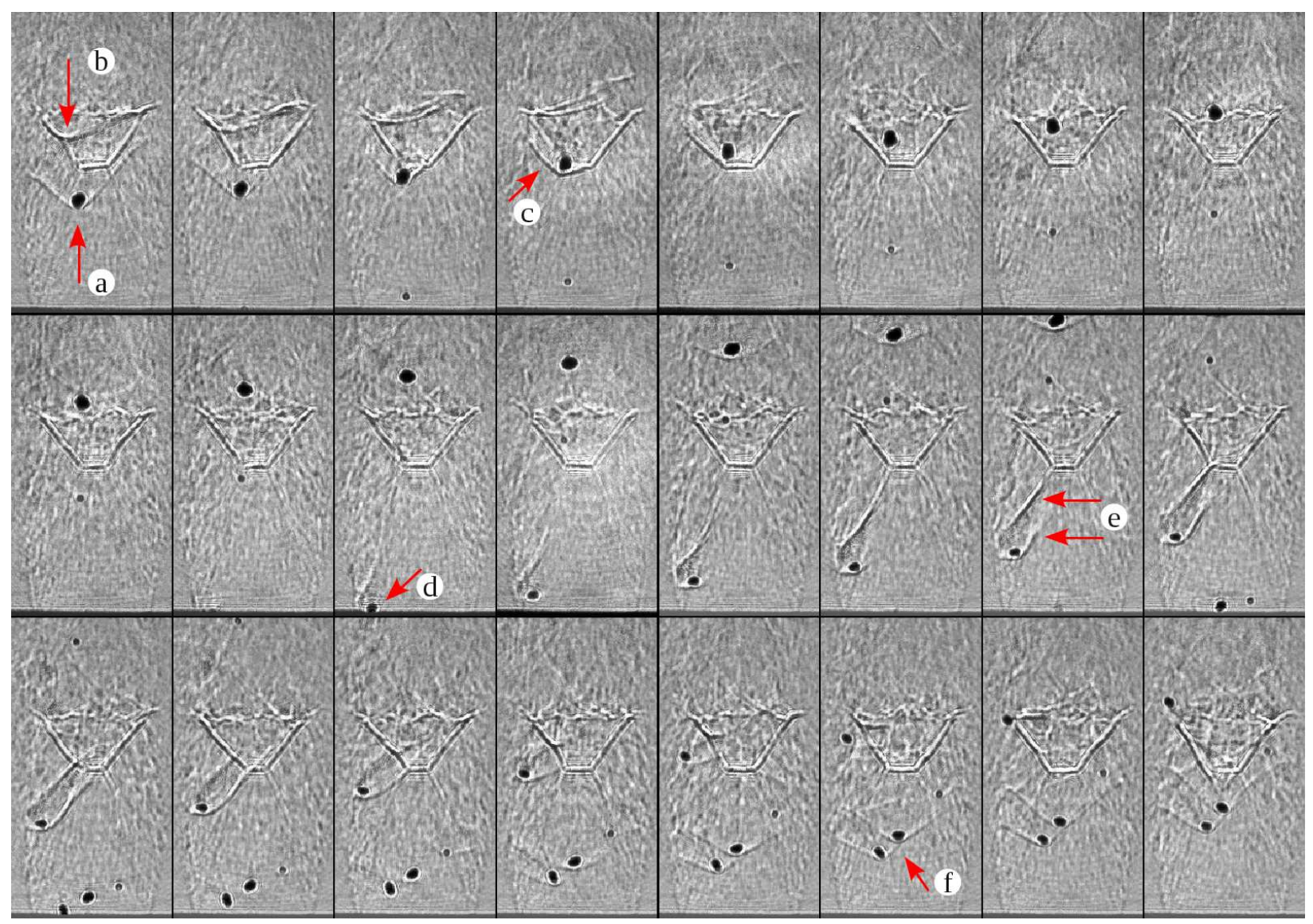

Figure 5. Visualization of particle-shockwave interaction recorded at $0.5 \mathrm{MHz}$ for a jet with $\mathrm{NPR}=5.0$ and $d_{p}=120 \mu \mathrm{m}$ particles

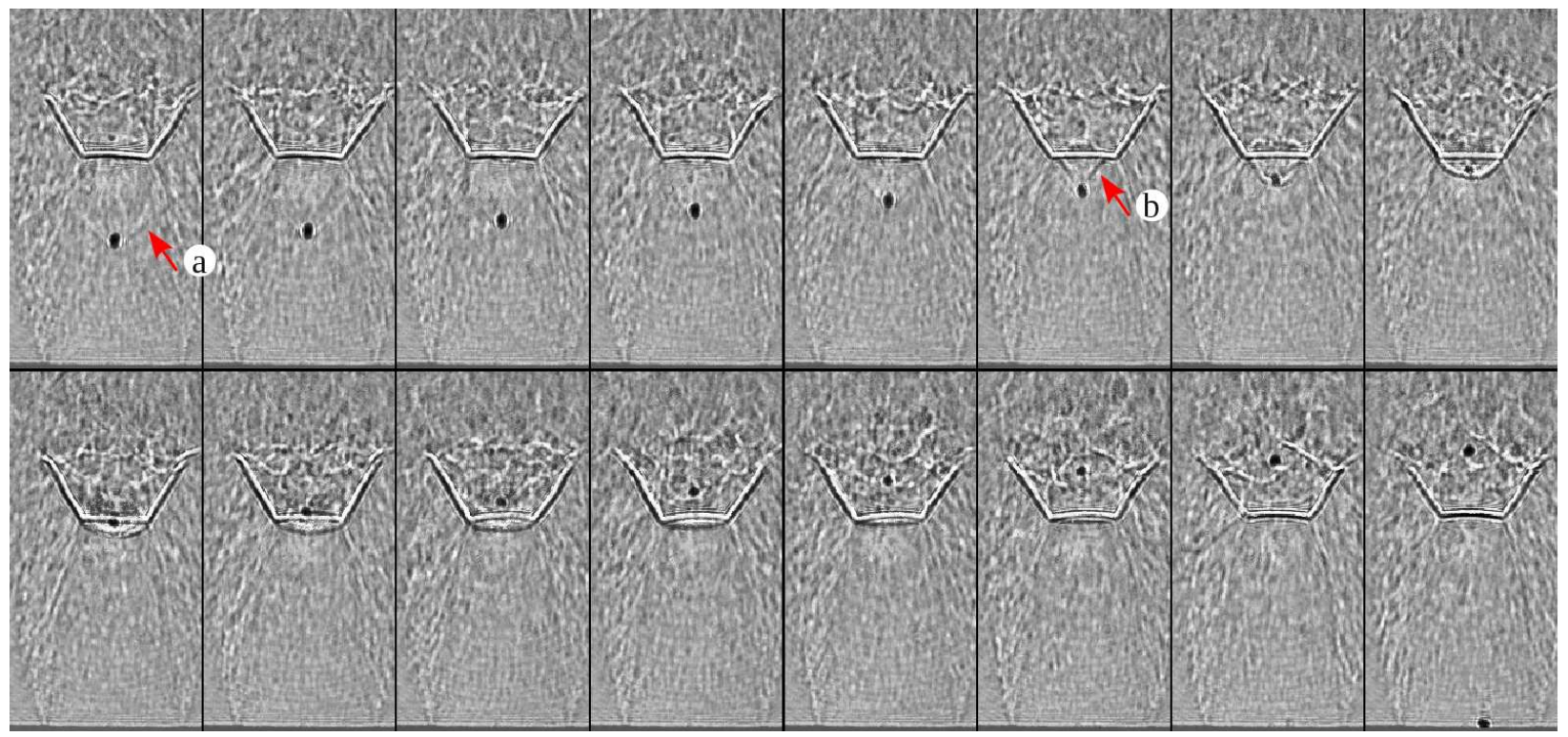

Figure 6. Visualization of particle-shockwave interaction recorded at $1.0 \mathrm{MHz}$ for a jet with NPR $=6.5$ and a $d_{p}=120 \mu \mathrm{m}$ particle 
reconstruct the optical field at a given depth position from the recorded hologram. ${ }^{7}$ In order to determine the depthwise position of an object, a focus function must be applied to quantify the sharpness of the reconstructed images. ${ }^{5,8}$ In the case of non-planar objects, such as particles in a jet, an in-plane $(x, y)$ interrogation area (IA) must be created for each particle. The focus function is then applied to each IA, and the depthwise position of the object is determined from the minimum or maximum value of the focus function.

In the present work five different focus functions have been tested, in order to evaluate which is the best suited for the current conditions. In the following the reconstructed intensity field of an IA is denoted $I(x, y ; z)$, were $z$ is the depthwise coordinate of the reconstructed plane. The five focus functions used here are defined as follows

$$
\begin{aligned}
\operatorname{SUM}(z) & =\sum_{x, y} I(x, y ; z), \text { for all } I(x, y ; z)>I_{0} \\
\operatorname{STD}(z) & =\sqrt{\frac{1}{N_{x} N_{y}} \sum_{x, y}(I(x, y ; z)-\bar{I}(z))^{2}}, \text { for all } I(x, y ; z)>I_{0} \\
\operatorname{GRA}(z) & =\sum_{x, y}|\nabla I(x, y ; z)| \\
\operatorname{LAP}(z) & =\sum_{x, y}\left(\nabla^{2} I(x, y ; z)\right)^{2} \\
\operatorname{VAR}(z) & =\frac{1}{N_{x} N_{y}} \sum_{x, y}(I(x, y ; z)-\bar{I}(z))
\end{aligned}
$$

where $I_{0}$ is a given threshold intensity and $\bar{I}(z)$ is the mean intensity of the IA. In words, the SUM function takes the sum of intensities above the threshold value $I_{0}$ for each $z$-position. A similar function was used in the work by Nguyen et al. ${ }^{4}$ The STD function calculates the standard deviation of the intensities above the threshold, this function was used in the work by Palero et al. ${ }^{6}$ The GRA function calculates the sum of intensity differences and is directly linked to the image contrast. The LAP function calculates the sum of differences of the intensity gradient and attains large values for images with high frequency edges. The VAR function calculates the variance of the intensities of the IA and is thus also closely linked to the image contrast. The GRA, LAP and VAR functions were compared by Choi \& Lee ${ }^{8}$ for a microscopic digital in-line holography setup. They found that the LAP function resulted in the best estimates of the depthwise position.

In the present setup, the focus functions attains close to constant values with significant noise artifacts when the object is nearly in focus. In order to increase the accuracy of the method a peak fit is made to the focus functions. Both a second order polynomial and a Gaussian function is tested for the peak fitting procedure.

\section{IV.A. Tests with planar targets}

In order to investigate the accuracy of the method for determining the depthwise object positions, tests are carried out using planar targets. For the first test, the $10 \times 10 \mathrm{~mm}$ calibration grid is used. The calibration grid is mounted on a small micrometer controlled traverse. Using the traverse, the grid is positioned in the focal plane of the imaging system and a series of shadow images are recorded. The grid is then moved away from the camera so that the focal plane is located $10.0 \mathrm{~mm}$ behind the grid and a series of holograms are recoded. To simulate the measurement situation, both the image and hologram series are recorded at $0.5 \mathrm{MHz}$ with an exposure time of $0.25 \mu \mathrm{s}$. Using the code by Ellenrieder and Soria, ${ }^{9}$ the intensity fields are reconstructed in a volume of $\pm 5 \mathrm{~mm}$ around the expected grid position in steps of $0.1 \mathrm{~mm}$, resulting in 101 reconstructed planes.

To establish where in the reconstructed space the grid is in focus, a cross-correlation is carried out between the reconstructed images and the recorded shadow image of the grid. The maximum correlation is found to be $0.3 \mathrm{~mm}$ in front of the physical grid position. This small discrepancy is well within the positional uncertainty of the physical grid. Figure 7 shows the recorded shadow image of the grid, the corresponding hologram and the reconstructed image at the grid plane. Figure 8 shows five slices through the reconstructed 


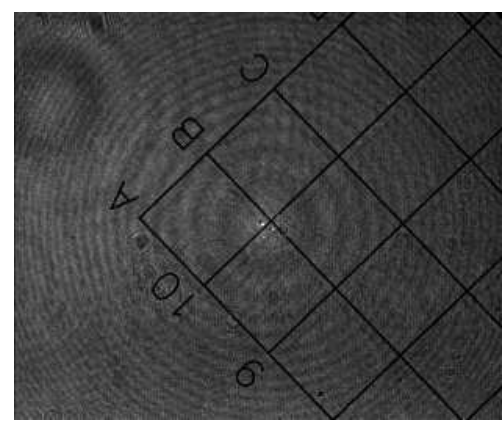

(a) Shadow Image

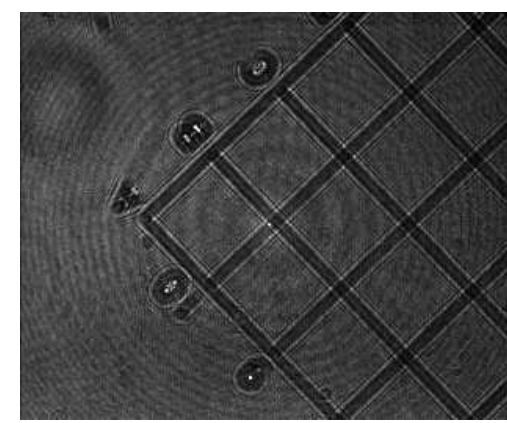

(b) Hologram

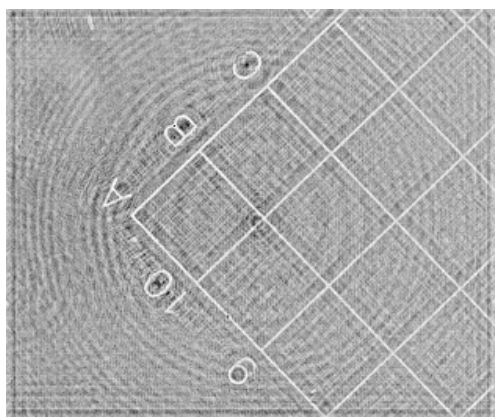

(c) Reconstructed intensity

Figure 7. Holography test, grid case.

3D intensity field. The 3D intensity field is centered around grid plane, denoted $z^{\prime}=0$. It is observed that the reconstructed images near the grid plane are characterized by sharp and well defined edges and have a low background intensity.

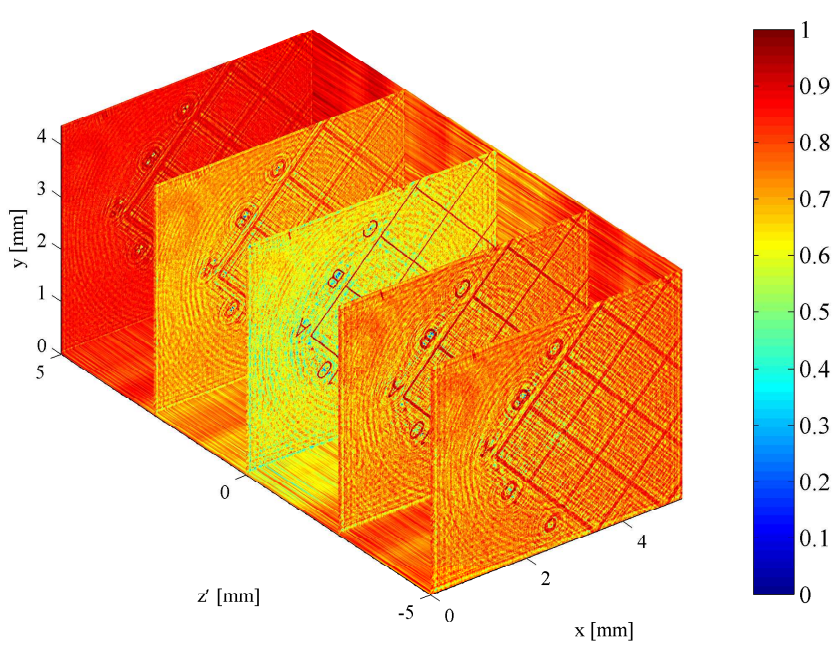

Figure 8. Reconstructed three dimensional intensity field. Note that the grid is in focus close to $z /=0.0 \mathrm{~mm}$

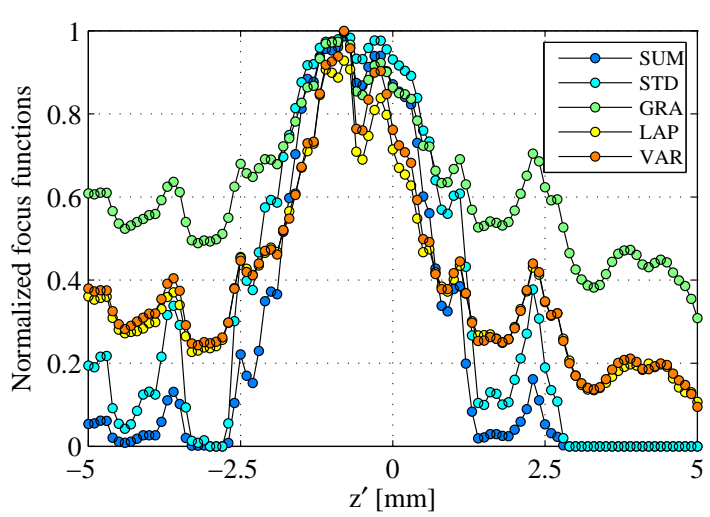

Figure 9. Depthwise profiles of the focus function values. The profiles are the ensamble average for the 21 IAs

21 IAs of $10 \times 10$ pixels are then created based on an intensity threshold value. The five focus criteria are applied to the IAs using both the polynomial and Gaussian peak fitting procedure. The depthwise profiles of the focus function values are presented on figure 9. The profiles show the ensemble average values of the 21 IAs. It is noted that the SUM function has the highest $S / N$ ratio and the GRA functions has the lowest.

On Figure 10 the mean depthwise positions obtained from the different focus functions are presented. From the plot it is observed that the depthwise position of the grid is successfully determined, with a maximum error of $0.2 \mathrm{~mm}$. The SUM and STD functions combined with the polynomial peak fitting, result in the best mean estimate and the smallest amount of scatter between estimates from different IAs.

In the second test case a new planar target is created by placing $d_{p}=120 \mu \mathrm{m}$ particles on a glass plate. Similarly to the grid test both shadow images and holograms are recorded. The recorded shadow image, hologram and reconstructed image at the target plane is presented on figure 11. The maximum correlation between the shadow image and the reconstructed images is again found to be very close to the target position, with a discrepancy smaller than the uncertainty of the target-plane position. In contrast to the grid test, the background intensity of the reconstructed images close to the target position is found to be relatively high (see Figure 12). Furthermore, reconstructed images with low background intensity are observed for planes approximately $2.5 \mathrm{~mm}$ behind the target. It is found that determination of the depthwise position of the test target using focus functions, is not possible in the particle case as the depthwise profile of the focus functions does not show a distinct peak as in the grid case(see Figure 13). It is not fully understood 


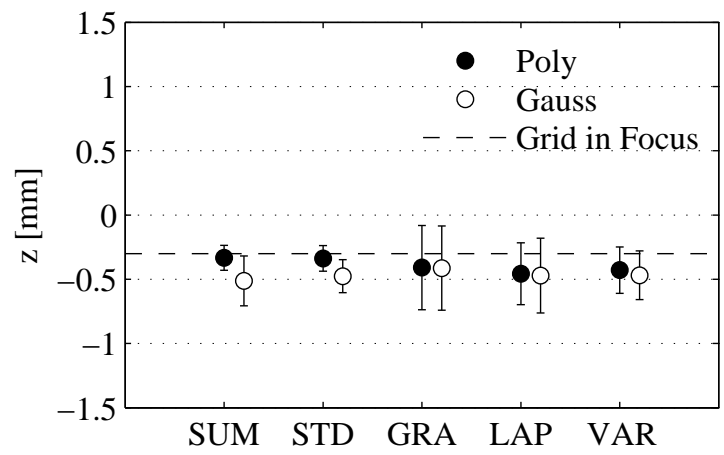

Figure 10. Comparison of estimated depthwise grid position using different focus criteria. The error bars correspond to a $95 \%$ confidence interval for a single estimate

why the reconstructed images with low background intensity occur at positions far from the target plane. One possible explanation is that they are noise artifacts caused by the relatively low spatial resolution of the imaging system.

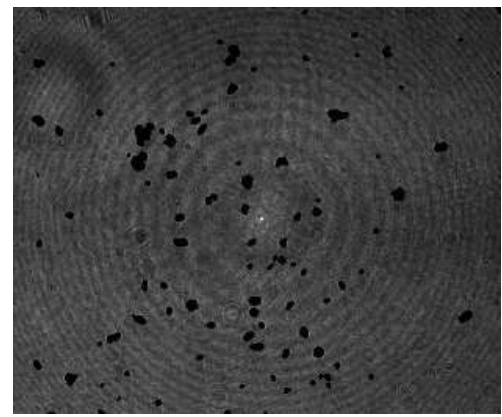

(a) Shadow image

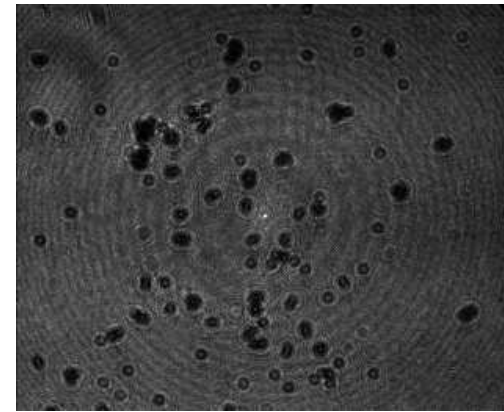

(b) Hologram

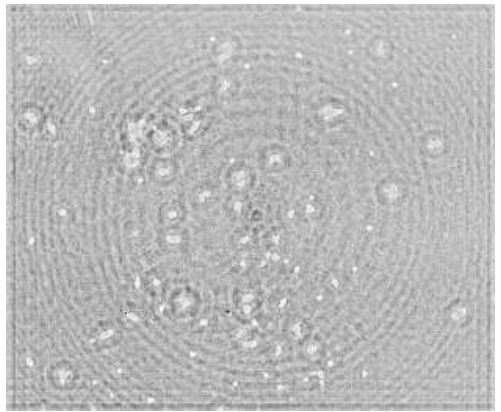

(c) Reconstructed intensity

Figure 11. Holography test, particle case.

\section{Summary}

Particle-fluid interactions in supersonic flows are relevant in many different applications e.g. the cold gasdynamic spray process. The cold gas-dynamic spray process has several advantages compared to conventional coating techniques, however, the optimal application of the process is hindered by a lack of understanding of the particle-fluid interactions. To obtain detailed information on the particle-fluid interactions in these high-speed flows special high performance techniques are required. The present work is an investigation into the applicability of magnified digital in-line holography with ultra-high-speed recording for the study of three-dimensional supersonic particle-laden flows.

An optical setup for magnified digital in-line holography is created, using an ultra-high-speed camera capable of frame rates of up to $1.0 \mathrm{MHz}$. To test the new technique an axisymmetric supersonic underexpanded particle-laden jet is investigated. The results show that the new technique allows for the acquisition of both qualitative data (visualizations) and quantitative data (in-plane particle tracking 2D-2C, not included in the paper) on the fluid and particle dynamics. The acquired visualizations show the time resolved interactions of particles and shock structures. It is among other things shown how even single particles can significantly perturb the shock structures of the jet.

In the second half of the paper, the potential for determining the three-dimensional particle positions using holographic reconstruction is investigated. In order to obtained the depthwise object position focus functions quantifying the image sharpness are applied to interrogation areas of the reconstructed images. Five focus functions are tested using two different planar targets. The first target is a $10 \times 10 \mathrm{~mm}$ calibration grid and the second target is $120 \mu \mathrm{m}$ particles on a glass plate. In the case with the calibration grid it is found that accurate location of the depthwise position of the target is possible. It is furthermore found 


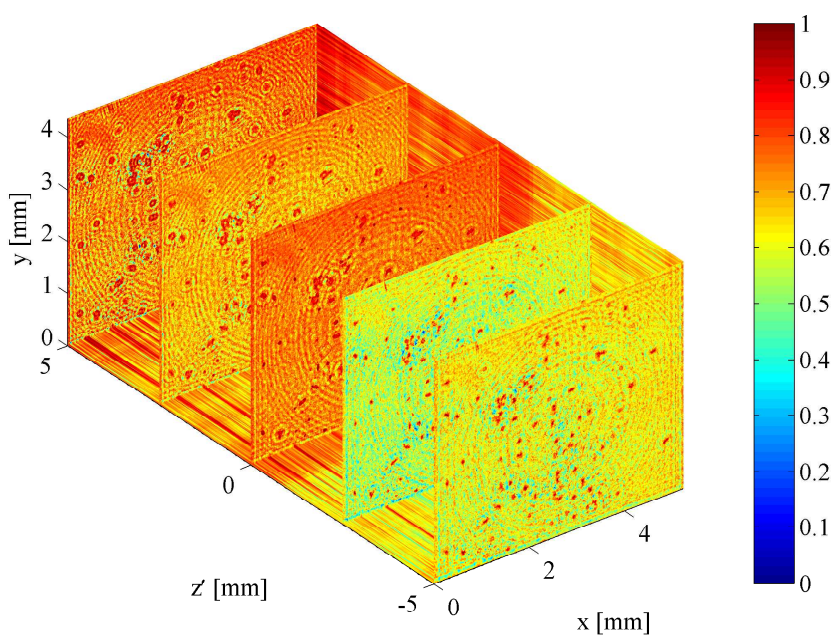

Figure 12. Reconstructed three dimensional intensity field. Note that the particles are in focus close to $z^{\prime}=0.0 \mathrm{~mm}$

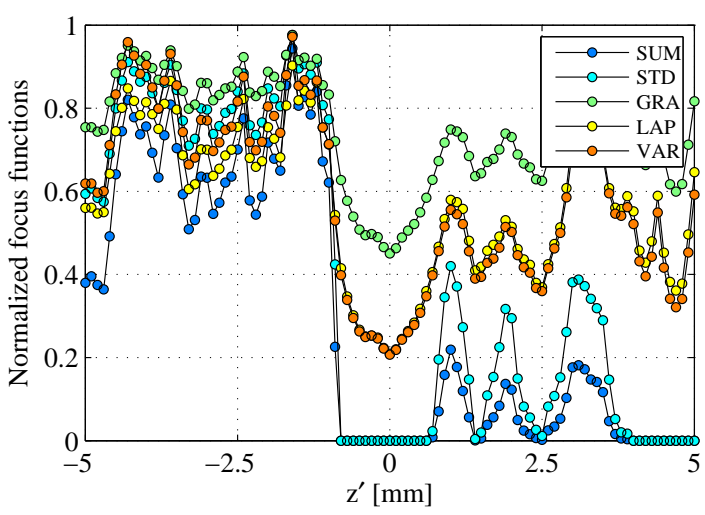

Figure 13. Depthwise profiles of the focus function values. The profiles are the ensamble average of IAs

that a focus function based on the sum of intensities above a threshold value gives the best results under the present conditions. When applying the same technique to the particle target, significant problems are encountered. It is expected that the problems are related to the relative low spacial resolution of the imaging system.

\section{Acknowledgments}

Financial funding from the Australian Research Council and the Danish Agency for Science Technology and Innovation (Grants No. 09-070608, MAN Diesel \& Turbo) is greatly acknowledged.

\section{References}

\footnotetext{
${ }^{1}$ Forney, L. J., "Particle impaction in axially symmetric supersonic flow," Aerosol Sci. Tech., Vol. 15, 1991, pp. 49-59.

${ }^{2}$ Quinlan, N., Kendall, M., Bellhouse, B., and Ainsworth, R., "Investigations of gas and particle dynamics in first generation needle-free drug delivery devices," Shock Waves, Vol. 10, 2001, pp. 395-404.

${ }^{3}$ Papyrin, A., Kosarev, V., Klinkov, S., Alkimov, A., and Fomin, V., Cold Spray Technology, Elsevier, 2007.

${ }^{4}$ Nguyen, D., Honnery, D., and Soria, J., "Measuring evaporation of micro-fuel droplets using magnied DIH and DPIV," Exp. Fluids, , No. 50, 2011, pp. 949-959.

${ }^{5}$ Sheng, J., Malkiel, E., and Katz, J., "Digital holographic microscope for measuring three-dimensional particle distributions and motions," Appl. Optics, Vol. 45, 2006, pp. 3893-3901.

${ }^{6}$ Palero, V., Arroyo, M. P., and Soria, J., "Digital holography for micro-droplet diagnostics," Exp. Fluids, , No. 43, 2007, pp. 185-195.

${ }^{7}$ Schnars, U. and Jueptner, W., Digital Holography, Springer-Verlag, 2005.

${ }^{8}$ Choi, Y.-S. and Lee, S.-J., "Three-dimensional volumetric measurement of red blood cell motion using digital holographic microscopy," Appl. Optics, Vol. 48, No. 16, 2009, pp. 2983-2990.

${ }^{9}$ Ellenrieder, K. V. and Soria, J., "Experimental measurements of particle depth of field in digital holography," In International Workshop on holographic metrology in fluid mechanics., May 2008.
} 